\title{
Coincidence echo statistics
}

\author{
Kenneth G. Foote \\ Institute of Marine Research, 5024 Bergen, Norway
}

(Received 11 May 1995; accepted for publication 8 September 1995)

Two scatterers at similar range give an echo which may appear to be due to a single scatterer. Methods for determining target strength that depend on resolving single scatterers may fail in this instance. Statistics associated with the described special case of coincidence are derived and illustrated by theoretical computation for the SIMRAD EK500 echo sounder system with the ES38B split-beam transducer resonant at $38 \mathrm{kHz}$. Connections to angle measurement in radar and swath bathymetry and to bottom-scattering-strength measurement are noted. (C) 1996 Acoustical Society of America.

PACS numbers: 43.30.Sf, 43.30.Gv, 43.20.Fn

\section{INTRODUCTION}

Many methods used to measure fish target strength in situ depend on resolution of single-target echoes. These include, for example, indirect methods, in which the effect of beam pattern is removed statistically, ${ }^{1}$ and direct methods, for example, those of dual beams and split beams, in which the beam pattern effect is removed by means of phase measurement with multiple beams. ${ }^{2}$

It is generally appreciated that single-target selection criteria must be used with care, if not great care, to avoid effects due to the presence of multiple targets at similar ranges. A practical illustration of the effect of selection criteria on the resultant target strength distribution is obtained by changing the acceptance limits for echo length. Increasing the upper limit often increases the registration of large targets, while decreasing the same may radically decrease both the number and magnitude of accepted echoes. This illustration becomes vivid when fish are loosely concentrated, as during the process of nighttime dispersion. Dual-beam or split-beam echo sounding systems, with so-called target strength analyzers, generally continue to deliver target strength data whatever the state of concentration.

The interesting question thus arises as to the effect of multiple targets with coincident or near-coincident echoes, referred to forthwith simply as coincident echoes, on the apparent single-target target strength distribution. It is remarked at the outset that this problem has important antecdents both in radar and in swath bathymetry. For radars used in so-called very-high-precision tracking applications, multiple targets are known to be a source of error. ${ }^{3}$ A multiple target may consist, for example, of two or more facets on the same target, differing in angle or range with respect to the transmitting or receiving antenna, or of different targets, such as two unresolved aircraft flying in formation. It is appreciated that the apparent angular location or range of the target may be outside the physical region of the target.

In swath bathymetry, interference due to multiple targets is recognized to be the major cause of depth error. ${ }^{4}$ Multiple targets located at the same range on or in the bottom may cause a phase sample dispersion, ${ }^{5}$ which is exactly analogous to the angle error or "glint" in the mentioned radar application. It may be significant that none of the methods described or developed by Masnadi-Shirazi et al. ${ }^{6}$ is able to resolve ambiguities due to multiple targets at the same range but different angles or same angle but different ranges, suggesting the fundamental nature of the problem.

In the present problem, the object of interest is the apparent target strength, determined in part by the angle error. This contrasts with the problem of angle error in radar and swath bathymetry applications, where the object of interest is the angle error per se. It is consequently necessary to specify a particular method for determining target strength. For definiteness in numerical computation, this is assumed to be the target strength analyzer in the SIMRAD EK500 echo sounder system with split-beam transducer resonant at 38 $\mathrm{kHz} .^{7}$

\section{THEORY}

\section{A. Beam pattern of a transducer aperture}

The transducer is defined as a shaded planar array of identical square elements. A subset of the elements defines an aperture. For the particular aperture $A$, the beam pattern amplitude factor in the direction $\hat{k}$ is

$$
D_{\mathrm{A}}(\hat{k})=\sum_{j \in \mathrm{A}} w_{j} \int_{A} \exp (i \mathbf{k} \cdot \mathbf{r}) d A_{j} / \sum_{j^{\prime} \in \mathrm{A}} w_{j^{\prime}},
$$

where $\mathbf{k}$ is the wave vector, $\hat{k}=\mathbf{k} / k, w_{j}$ is the amplitude weight of the $j$ th transducer element, and $\mathbf{r}$ is the position of the differential surface element $d A_{j}$. In rectangular coordinates, $\hat{k}=(\sin \theta \cos \phi, \sin \theta \sin \phi, \cos \theta)$. Referring $\mathbf{r}$ to the center $\mathbf{r}_{j}$ of the $j$ th transducer element, and integrating over the area,

$$
D_{\mathrm{A}}(\hat{k})=D_{1}(\hat{k}) \sum_{j \in \mathrm{A}} w_{j} \exp \left(i \mathbf{k} \cdot \mathbf{r}_{j}\right) / \sum_{j^{\prime} \in \mathrm{A}} w_{j^{\prime}},
$$

where $D_{1}=\operatorname{sinc}(k a \sin \theta \cos \phi) \operatorname{sinc}(k a \sin \theta \sin \phi) \quad$ is the beam pattern amplitude factor of a single, square array element of side length $a, \operatorname{sinc}(x)=\sin (x) / x$.

\section{B. Echo amplitude due to multiple targets at similar range}

The echo pressure resulting from ensonification of an ensemble of targets can be expressed by the equation

$$
p=\sum p_{j} s_{j}\left(t-2 r_{j} / c\right),
$$


where $p_{j}$ is the echo amplitude due to the $j$ th target, $s_{j}$ is the corresponding echo waveform, $r_{j}$ is the range of the $j$ th target, and $c$ is the speed of sound. For the narrow-band operation implicit in use of a resonant transducer, the individual echo waveforms $s_{j}$ are essentially identical, say, to the waveform

$$
s(t)=\exp \left(i \omega_{0} t\right) \operatorname{rect}\left(\frac{t-\tau / 2}{\tau}\right),
$$

where $\omega_{0}=c k$ is the angular frequency at resonance, $\tau$ is the signal duration, and $\operatorname{rect}(x)=1$ for $|x| \leqslant \frac{1}{2}$ and 0 for $|x|>\frac{1}{2}$. For targets at similar, sufficiently large ranges in the transducer far field, differing by no more than one-half wavelength, $\lambda / 2$, then to a very good approximation and to within a constant of proportionality,

$$
p_{j}=b_{j} \sigma_{j}^{1 / 2},
$$

where $b_{j}=D_{T j} D_{R j}, D_{T j}$ and $D_{R j}$ are the beam pattern amplitude factors of the respective transmit and receive apertures in the direction of the $j$ th target, and $\sigma_{j}$ is the backscattering cross section of the same. Substituting in Eq. (3) and ignoring common factors,

$$
p=\sum b_{j} \sigma_{j}^{1 / 2} \exp \left(i \psi_{j}\right)
$$

where $\psi_{j}$ is the phase associated with the $j$ th target, namely $4 \pi r_{j} / \lambda$.

In the special case of just two targets, which is the one considered here, Eq. (5) can be simplified. To within a constant phase factor,

$$
p=b_{1} \sigma_{1}^{1 / 2}+b_{2} \sigma_{2}^{1 / 2} \exp (i \chi),
$$

where $\chi$ is the relative phase.

It is noted here that for the case of $n=2$ targets, a range of variation in $\psi_{j}$ in Eq. (5) of $\lambda / 4$ is sufficient to encompass the entire range of possible interferences. In the more general case of $n>2$ targets, a range of variation in $\psi_{j}$ of $\lambda / 2$ is sufficient, as is immediately evident by construction of phasor diagrams.

\section{Split-beam echo processing}

A split-beam transducer is electrically divided into quadrants. When transmitting, all quadrants are excited simultaneously, forming a single beam. When receiving, each quadrant acts independently to generate its own received echo signal. Half-beams are formed, and the phase difference between fore-and-aft halves and port-and-starboard halves detected. Knowing these two angles, hence target direction, the beam pattern is known a priori. The effect of the beam pattern on the sum-beam echo amplitude can thus be removed, resulting in an estimate for $\sigma_{j}$. A mathematical description follows.

For the assumed complex echo pressure $p$,

$$
p=|p| \exp \left\{i \tan ^{-1}[\operatorname{Im}(p) / \operatorname{Re}(p)]\right\} .
$$

The phase is $\tan ^{-1}[\operatorname{Im}(p) / \operatorname{Re}(p)]$. The quadrants of the transducer are numbered sequentially from the forward starboard quarter (1), to forward port (2), to aft port (3), to aft starboard (4). The result of combining the echo pressure reg- istered by quadrants 1 and 2 is the half-beam $h_{12}$, and so forth. The angle of the target relative to the transducer in the fore-and-aft plane is determined from the direction cosine

$$
\begin{aligned}
\alpha= & \mathrm{S}^{-1}\left\{\tan ^{-1}\left[\operatorname{Im}\left(h_{12}\right) / \operatorname{Re}\left(h_{12}\right)\right]\right. \\
& \left.-\tan ^{-1}\left[\operatorname{Im}\left(h_{34}\right) / \operatorname{Re}\left(h_{34}\right)\right]\right\},
\end{aligned}
$$

where $\mathrm{S}$ is the so-called angle sensitivity factor, which is used to convert the phase difference to a spatial angle. The factor $\mathrm{S}$ is approximately equal to $k d$, where $d$ is the effective distance between the transducer halves. The angle of the target in the port-and-starboard plane is determined from the direction cosine

$$
\begin{aligned}
\beta= & \mathrm{S}^{-1}\left\{\tan ^{-1}\left[\operatorname{Im}\left(h_{14}\right) / \operatorname{Re}\left(h_{14}\right)\right]\right. \\
& \left.-\tan ^{-1}\left[\operatorname{Im}\left(h_{23}\right) / \operatorname{Re}\left(h_{23}\right)\right]\right\} .
\end{aligned}
$$

Equations (8) and (9) apply in the usual small-angle limit.

In a rectangular coordinate system with origin at the transducer center, $x$ axis pointing to starboard, $y$ axis forward, and $z$ axis downward,

$$
\begin{aligned}
& \alpha=\hat{k} \cdot \hat{y}=\sin \theta \sin \phi, \\
& \beta=\hat{k} \cdot \hat{x}=\sin \theta \cos \phi ;
\end{aligned}
$$

hence

$$
\begin{aligned}
& \theta=\sin ^{-1}\left(\alpha^{2}+\beta^{2}\right)^{1 / 2}, \\
& \phi=\tan ^{-1}(\alpha / \beta) .
\end{aligned}
$$

That is, the target position can be identified in ordinary polar coordinates based on measurement of half-beam phase differences, with immediate computation of $\alpha$ and $\beta$.

Two targets at similar range but generally different angular locations $\left(\theta_{1}, \phi_{1}\right)$ and $\left(\theta_{2}, \phi_{2}\right)$ will produce echoes that appear to be due to a single scatterer at a third location $(\theta, \phi)$. If this lies in the main lobe of the split-beam transducer, it will, under the stated condition of similar range, be perceived as a single scatterer, and compensation for the apparent beam pattern loss accordingly applied. Larger apparent target angles are rejected. A series of measurements in the presence of multiple targets will thus in general produce a distribution of apparent single-target target strengths, at least some of which are spurious.

\section{COMPUTATIONAL METHOD}

In order to investigate the effect of multiple targets on the target strength distribution derived by means of splitbeam processing, the two-target case is considered according to the following model.

\section{A. Split-beam transducer}

For definiteness, this is assumed to be the SIMRAD ES38B transducer. This is a truncated square array of identical square elements of side length $30 \mathrm{~mm}$ and center-tocenter distance along rows and columns of $32 \mathrm{~mm}$, with 


$\begin{array}{rrrrr}100 & 100 & 70 & & \\ 100 & 100 & 70 & 70 & \\ 100 & 100 & 100 & 70 & 70 \\ 100 & 100 & 100 & 100 & 100 \\ 100 & 100 & 100 & 100 & 100\end{array}$

FIG. 1. Amplitude weights of elements in the forward starboard quadrant of the SIMRAD ES38B transducer.

an operating resonant frequency of $38 \mathrm{kHz}$. The amplitude weights in the forward starboard quadrant are shown in Fig. 1.

\section{B. Transducer angle sensitivity factor}

The nominal figure given by the manufacturer is $21.9 .^{8}$ For the sound speed assumed here, namely $1470.6 \mathrm{~m} / \mathrm{s}$, defined by temperature $5^{\circ} \mathrm{C}$, salinity $35 \mathrm{ppt}, p \mathrm{H} 8.8$, and depth $0 \mathrm{~m},{ }^{9}$ this factor was determined by the following procedure. For an assumed value of $S$ and given target position, the four half-beams are computed, followed by computation of the direction cosines in Eqs. (8) and (9). Computation of $\theta$ and $\phi$ by Eq. (11) allows the beam pattern compensation value to be determined. Comparison with the beam pattern value at the given target position allows the beam pattern compensation error to be gauged. Repetition of this computation for each target position over a grid of points uniformly covering the transducer beam cross section allows the mean beam pattern compensation error to be determined. The described computations are repeated for a range of values of $\mathrm{S}$. The value that produces no mean beam pattern compensation error is $S=23.2$. Use of this value ensures that the compensation method does not introduce a bias into the mean backscattering cross section of a single target.

\section{Spatial distribution}

The target range is assumed to be constant and equal for the two targets to within one-quarter of the acoustic wavelength. The targets are assumed to be distributed with equal probability of occurrence anywhere in the cross section of the transducer beam within the -6 -dB level, i.e., within the angular zone of acceptance for split-beam processing. For the ES38B transducer and medium sound speed of 1470.6 $\mathrm{m} / \mathrm{s}$, this zone is defined by a limiting polar angle that is to a fair approximation $4.66 \mathrm{deg}$.

\section{Target strength distributions}

Each of two distributions is considered through the probability density function of target strength TS.

(1) Constant target strengths: The respective target strength distributions are

$$
f_{1}(\mathrm{TS})=\delta(\mathrm{TS})
$$

and

$$
f_{2}(\mathrm{TS})=\delta(\mathrm{TS}+\Delta \mathrm{TS}),
$$

where $\delta$ is the Dirac delta function, and $\Delta \mathrm{TS}$ is the constant difference in TS.

(2) Normally distributed target strengths: Both target strengths independently follow the same normal distribution, namely,

$$
f(\mathrm{TS})=\left(2 \pi s^{2}\right)^{-1 / 2} \exp \left[-(\mathrm{TS}-\overline{\mathrm{TS}})^{2} /\left(2 s^{2}\right)\right],
$$

where $\overline{\mathrm{TS}}$ and $s$ denote the respective mean and standard deviation.

\section{E. Echo amplitude}

The two-target form is computed according to Eq. (6), where $\sigma=4 \pi 10^{(\mathrm{TS} / 10)}$, and $\chi$ is uniformly distributed over $\pi$ rad. That is,

$$
f(\chi)=\pi^{-1},
$$

where $0 \leqslant \chi \leqslant \pi$.

\section{F. Simulation of split-beam processing}

In addition to computing the sum beam for use with Eq. (6), the quadrant-beam responses are also computed. Halfbeams are computed, assuming $\mathrm{S}=23.2$, and the alongships and athwartships angles computed according to Eq. (10). Use of the resulting values in Eq. (11) determines the apparent single-target position $(\theta, \phi)$, thence the sum-beam compensation factor $\hat{b}$ if within the limiting angle. For a single realization of the described stochastic model, then, the apparent backscattering cross section is

$$
\hat{\sigma}=\left|b_{1} \sigma_{1}^{1 / 2}+b_{2} \sigma_{2}^{1 / 2} \exp (i \chi)\right|^{2} / \hat{b}^{2},
$$

and the corresponding apparent target strength, for $\hat{\sigma}$ in SI units, is

$$
\widehat{\mathrm{TS}}=10 \log \frac{\hat{\sigma}}{4 \pi} \text {. }
$$

\section{G. Apparent target strength distribution}

Repeated exercise of the model determines a series of values for the apparent target strength. In this way the distribution $f(\mathrm{TS})$ is generated. When simulated on a digital computer, the values are sorted in contiguous TS bins of width $0.5 \mathrm{~dB}$.

\section{H. Numerical parameters}

By simulation, two targets are allowed to occupy a range of paired positions entirely covering the transducer beam cross section with equal probability of occurrence. This is done by systematic and uniform variation of the polar angles $\theta_{1}$ and $\theta_{2}$ in 50 equal increments $\Delta \theta$ over $4.66 \mathrm{deg}$. The azimuth $\phi_{1}$ is varied over the range $[0, \pi / 4]$ in six increments $\Delta \phi_{1}=\pi / 24$, and $\phi_{2}$ is moved over the range $\left[\phi_{1}, \phi_{1}+\pi\right]$ in 16 increments of size $\Delta \phi_{2}=\pi / 16$. The represented incremental area thus increases as $\sin \theta_{1} \sin \theta_{2} \Delta \theta_{1} \Delta \theta_{2} \Delta \phi_{1} \Delta \phi_{2}$. The phase $\chi$ is varied uniformly over the range $[0, \pi] \operatorname{rad}$ in 19 increments of size $\Delta \chi=\pi / 19$. In the first case of constant target strengths, these are applied directly. In the second case of normally distributed target strengths, these are indepen- 
dently drawn from the same distribution for each combination of values $\theta_{1}, \theta_{2}, \phi_{1}$, and $\phi_{2}$. A pseudorandom number generator of linear congruential type is employed, with simple realization on FORTRAN compiler f77 as implemented on SUN computers.

\section{RESULTS AND DISCUSSION}

Apparent single-target target strength distributions are shown in Fig. 2 for the case of constant target strengths and in Fig. 3 for the case of normally distributed target strengths.

In the case of the constant target strengths, shown in Fig. 2, the strongest effect is observed for equal target strengths, in Fig. 2(a). With decreasing signal-to-noise ratio (SNR) in Fig. 2(b)-(d), the effect of the second, weaker target strength is seen to be progressively less, evidently serving as a minor perturbation to the single-target distribution $f_{1}(\mathrm{TS})=\delta(\mathrm{TS})$. The results are further illustrated by the change in average backscattering cross section of the apparent single target. For the distributions shown in Fig. 2(a)-(d), the corresponding logarithmic measure is $2.04,0.91,0.52$, and $0.12 \mathrm{~dB}$, respectively.

In the case of the normally distributed target strengths, the resultant distributions of apparent target strength in Fig. 3 display characteristics that are consistent with those in Fig. 2 and which can be understood in their light. First, the distribution in Fig. 3(a) closely resembles that in Fig. 2(a), as indeed it should since the case of constant and equal target strengths can be viewed as the limiting case of a normal distribution with vanishing standard deviation. Second, the distribution of apparent target strength in Fig. 3(e), due to the single-target distribution $N(0,10)$, bears a closer resemblance to the original single-target distribution than do any of the others. With increasing dispersion, the chance of two values being drawn from the same distribution being very similar is small, while that of being quite different is large, hence explaining the smaller effect, as also observed in Fig. 2(d) compared to that in Fig. 2(a). The mean values of the apparent single-target distribution, as computed in the intensity or $\sigma$ domain, are $2.04,5.12,7.31,9.45$, and $15.33 \mathrm{~dB}$ for the five distributions arranged in order of increasing standard deviation. The corresponding values of the underlying single-target distribution are $0,0.11,1.00,2.75$, and 10.32 $\mathrm{dB}$, as this distribution is log-normal, with increasing bias with increasing width.

The several distributions and computations of average measures include only those echoes that survive the detected-angle selection criterion, namely, that $\theta$ not exceed $4.66 \mathrm{deg}$. In the cases represented by Fig. 2(a)-(d), the percentage of accepted echoes is $77.9 \%, 81.8 \%, 83.6 \%$, and $86.8 \%$, respectively. In the cases represented by Fig. 3(a)(e), the acceptance number is in the range $77.7 \%-79.3 \%$.

A detailed investigation identifies the nature of the rejection process for apparent single targets. When the quantities $b_{1} \sigma_{1}^{1 / 2}$ and $b_{2} \sigma_{2}^{1 / 2}$ in Eq. (6) are nearly equal, and the phase factor $\chi$ is close to $\pi$, the sum becomes small and the apparent phase angle unstable. Out-of-range values can then result. These are rejected if greater than the threshold angle 4.66 deg, but other, irregular values not exceeding the threshold
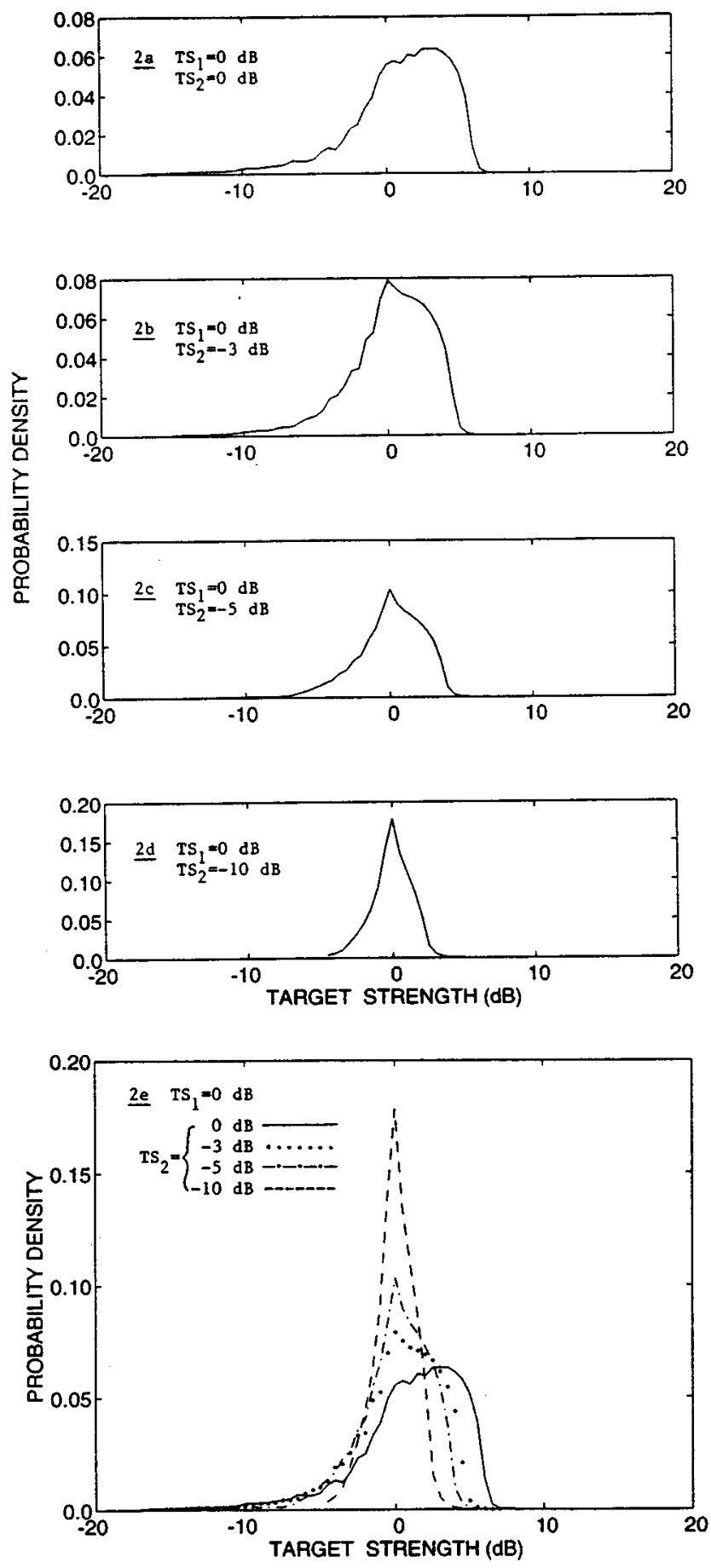

FIG. 2. Probability density functions of the apparent single-target target strength due to coincident echoes from two targets in the main beam of the SIMRAD ES38B split-beam transducer, with constant target strengths $\mathrm{TS}_{1}=0 \mathrm{~dB}$ and $\mathrm{TS}_{2}$ as indicated in parts (a)-(d). Part (e) is a superposition of parts (a)-(d) but with the same scale.

angle wreak their damage on the apparent target strength distribution.

The particular mean levels of target strength assumed in the computations do not limit the results. In fact, the constant value $\mathrm{TS}_{1}=0 \mathrm{~dB}$ assumed in the computations in Fig. 2 and mean distribution value $\overline{\mathrm{TS}}=0 \mathrm{~dB}$ assumed for Fig. 3 may be viewed as arbitrary references. The displayed distributions 

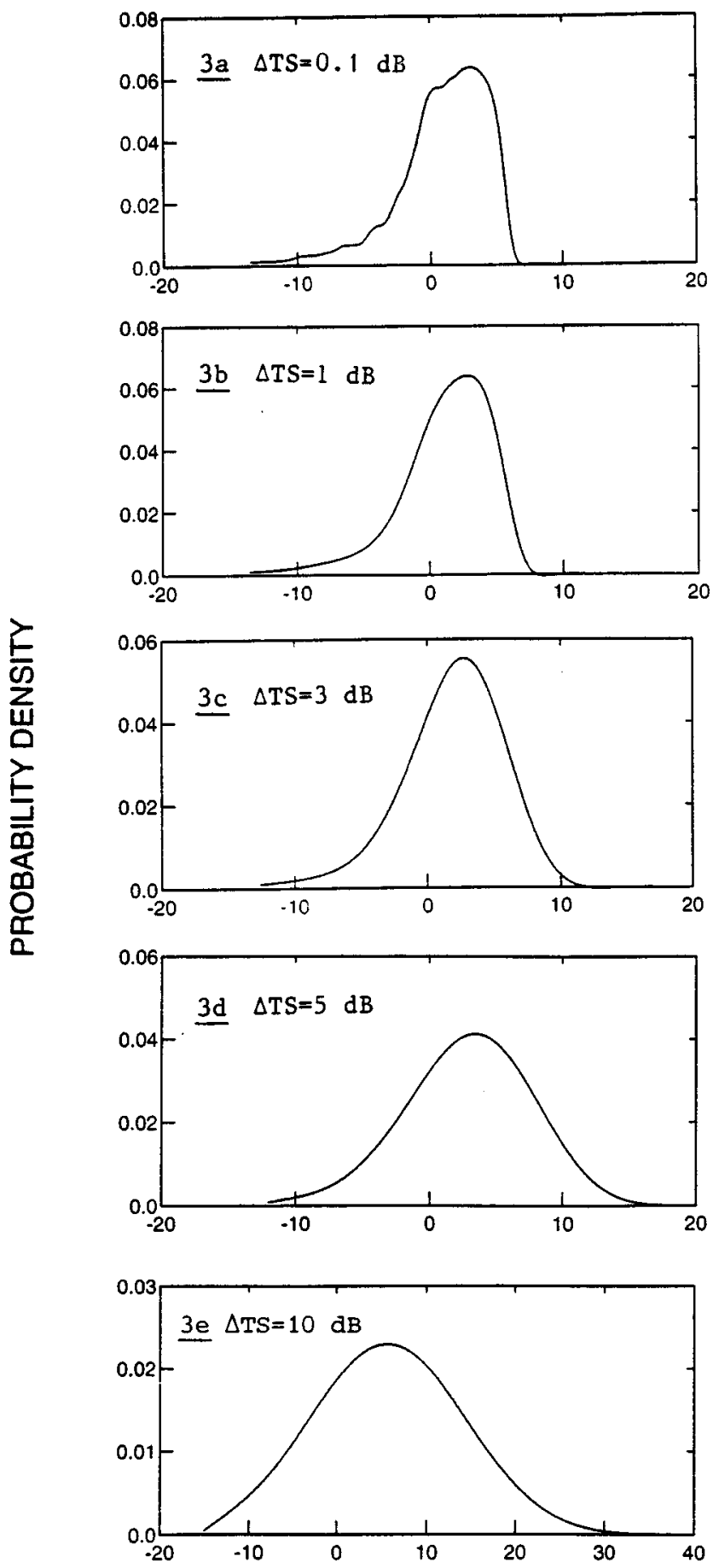

TARGET STRENGTH (dB)

FIG. 3. Probability density function of the apparent single-target target strength due to coincident echoes from two targets in the main beam of the SIMRAD ES38B split-beam transducer, with target strengths independently drawn from the same normal distribution of target strength, $N(\overline{\mathrm{TS}}, \Delta \mathrm{TS})$, where $\mathrm{TS}=0 \mathrm{~dB}$ and $\Delta \mathrm{TS}$ assumes the indicated values.

apply to other absolute levels by a simple translation in target strength domain.

In general, no matter what the application, simultaneous multiple-frequency measurements can help resolve situations of ambiguity. The phase is sensitive to frequency, so situa- tions of multiple scatterers will differentiate themselves from single-scatterer situations through frequency-dependent phases. If the apparent target position varies with frequency, it can be assumed to be due to the presence of multiple scatterers at similar range, hence rejected.

While the present study involves angle errors due to interference, a problem common to both radar target-tracking and swath bathymetry, results for the effect of angle error on target strength may be directly applied to problems in acoustic scattering by the sea bottom. Makris and Berkson present an empirical histogram of scattering strength with standard deviation of $7 \mathrm{~dB} .{ }^{10}$ Were this the underlying distribution of each of two interfering targets, the result of coincidence would be a broadening of the apparent single-target target strength distribution to a degree intermediate to those in Fig. 3(d) and (e), with standard deviations of 5 and $10 \mathrm{~dB}$. Direct measurements of bottom scattering strength, for example, of the type reported by Jackson et al., ${ }^{11}$ may be corrupted by angle errors due to coincident echoes. Methods for determining seafloor roughness that depend on measurement of echo strength, for example, Stanton's method, ${ }^{12}$ must also be affected by coincidences. Constructing maps of seafloor acoustic backscattering strength, mentioned by de Moustier, ${ }^{13}$ is a further process that would be sensitive to coincident echoes.

\section{CONCLUSIONS}

Clearly, the presence of multiple targets at similar range can change the character of a target strength distribution as determined with a split-beam echo sounder. Two consequences are broadening of the distribution and biasing of the average measure of target strength. The effect of digital signal processing on split-beam operation, not simulated here, is to produce a further, slight broadening of the distribution, but without significant bias.

While the present analysis aims to quantify the effects of coincidence in two-target echoes on target strength, as derived with a particular split-beam target strength analyzer, the effects are recognized to be common to other methods of target strength determination that depend on the resolution of single targets. Avoidance of multiple-target effects by operating only under unambiguous conditions of dispersion is the recommended practice.

\section{ACKNOWLEDGMENTS}

E. Ona is thanked for calling attention to the problem. Discussions with T. K. Stanton on the phenomenon of instability in detection angle of apparent single target are gratefully acknowledged. D. Chu, H. Nes, and D. Tang are thanked for information on other applications of the work. $\varnothing$. Østensen is thanked for assistance in preparing Fig. 3.

${ }^{1}$ K. G. Foote, "Summary of methods for determining fish target strength at ultrasonic frequencies," ICES J. Mar. Sci. 48, 211-217 (1991).

${ }^{2}$ J. E. Ehrenberg, "A comparative analysis of in situ methods for directly measuring the acoustic target strength of individual fish," IEEE J. Ocean. Eng. OE-4, 141-152 (1979).

${ }^{3}$ D. D. Howard, "Tracking radar," in Radar Handbook, edited by M. I. Skolnik (McGraw-Hill, New York, 1990), 2nd ed., Chap. 18. 
${ }^{4}$ P. N. Denbigh, "Swath bathymetry: principles of operation and an analysis of errors," IEEE J. Ocean. Eng. 14, 289-298 (1989).

${ }^{5} \mathrm{H}$. Matsumoto, "Characteristics of SeaMARC II phase data," IEEE J. Ocean. Eng. 15, 350-360 (1990).

${ }^{6}$ M. A. Masnadi-Shirazi, C. de Moustier, P. Cervenka, and S. H. Zisk, "Differential phase estimation with the SeaMARC II bathymetric sidescan sonar system," IEEE J. Ocean. Eng. 17, 239-251 (1992).

${ }^{7}$ H. Bodholt, H. Nes, and H. Solli, "A new echo-sounder system," Proc. IOA 11(3), 123-130 (1989).

${ }^{8} \mathrm{H}$. Bodholt, "Split-beam transducer for target strength measurement," in Scandinavian Cooperation Meeting in Acoustics XIII, edited by $\mathrm{H}$. Hobæk, Department of Physics Sci./Tech. Rep. 227 (University of Bergen, Bergen, Norway), p. 73.
${ }^{9} \mathrm{~K}$. V. Mackenzie, "Nine-term equation for sound speed in the oceans," $\mathrm{J}$. Acoust. Soc. Am. 70, 807-812 (1981).

${ }^{10}$ N. C. Makris and J. M. Berkson, "Long-range backscatter from the MidAtlantic Ridge," J. Acoust. Soc. Am. 95, 1865-1881 (1994).

${ }^{11}$ D. R. Jackson, A. M. Baird, J. J. Crisp, and P. A. G. Thomson, "Highfrequency bottom backscatter measurements in shallow water," J. Acoust. Soc. Am. 80, 1188-1199 (1986).

${ }^{12}$ T. K. Stanton, "Sonar estimates of seafloor microroughness," J. Acoust. Soc. Am. 75, 809-818 (1984).

${ }^{13} \mathrm{C}$. de Moustier, "Signal processing for swath bathymetry and concurrent seafloor acoustic imaging," in Acoustic Signal Processing for Ocean Exploration, edited by J. M. F. Moura and I. M. G. Lourtie (Kluwer Academic, Dordrecht, 1993), pp. 329-354. 